\title{
Dirac fermions in Som-Raychaudhuri space-time with scalar and vector potential and the energy momentum distributions
}

\author{
Parisa Sedaghatnia ${ }^{1, \mathrm{a}}$, Hassan Hassanabadi ${ }^{1, \mathrm{~b}}$, Faizuddin Ahmed $^{2, \mathrm{c}}$ \\ ${ }^{1}$ Faculty of Physics, Shahrood University of Technology, P. O. Box: 3619995161-316, Shahrood, Iran \\ ${ }^{2}$ Ajmal College of Arts and Science, Dhubri, Assam 783324, India
}

Received: 7 April 2019 / Accepted: 14 June 2019 / Published online: 25 June 2019

(C) The Author(s) 2019

\begin{abstract}
The main object of the present paper is to investigate the Dirac equation (Dirac fermions) in presence of scalar and vector potential in a class of flat Gödel-type space-time called Som-Raychaudhuri space-time by using the methods quasi-exactly solvable (QES) differential equations and the Nikiforov Uvarov (NU) form. In addition, we evaluate the Einstein, and the Papapetrou.
\end{abstract}

\section{Introduction}

Som and Raychaudhuri [1] provided a family of stationary cylindrically symmetric solutions of the Einstain-Maxwell equations corresponding to a charged dust distribution in rigid rotation. In this solution, the Lorentz force vanishes everywhere and the ratio of charge density to mass density may assume arbitrary value. Barrow and Dabrowski [2] showed that homogeneous Gödel-type space-times need not contain closed time-like curves in low-energy effective string theories. They found exact solutions for the Gödel-type metric in string theory [3] for the full $O(\alpha)$ action including both dilaton and axion fields $[2,4]$. The Som-Raychaudhuri space-time belongs to the class of flat Gödel-type solutions. Different aspects of the Gödel-type solutions are discussed in [5-8]. Gürses et al. showed with several examples that Gödeltype metrics can be used in obtaining exact solutions to various supergravity theories and in constructing space-times that contain both closed time-like and closed null curves, and that contain neither of these [9]. Clifton et al. [10] determined the general conditions for the existence of Gödel, Einstein static, and de-Sitter universes in gravity theories derived from a Lagrangian that is an arbitrary function of the scalar curvature and Riemann curvature invariants. Explicit expres-

\footnotetext{
a e-mail: pa.sedaghatnia@gmail.com

b e-mail: h.hasanabadi@shahroodut.ac.ir

c e-mail: faizuddinahmed15@gmail.com
}

sions for the solutions were found in terms of the parameters defining the Lagrangian. Also they determined the conditions on the Lagrangian of the theory under which time-travel is allowed in Gödel universes.

Several researchers have been investigated the physical properties of a series of backgrounds with the Som Raychaudhuri space-time. For example, properties of the rotating Som-Raychaudhuri homogeneous space-time, relativistic quantum dynamics of spin-zero particles in SomRaychaudhuri space-time under the influence of the gravitational field produced by a topology [11], the Klein-Gordon oscillator in Som-Raychaudhuri space-time with a cosmic dispiration [12], linear confinement of a scalar particle in Som-Raychaudhuri space-time [13], linear confinement of a scalar particle in a topologically trivial flat Gödel-type spacetime [14]. Additionally, in Ref. [15] the quantum dynamics of scalar and spin-half particles in Gödel-type space-times with positive, negative and zero curvatures, were investigated. In Refs. $[16,17]$ the quantum dynamics of scalar particles in a class of Gödel-type solutions, were investigated. They observed the similarity of the energy levels with the Landau problem in flat, spherical and hyperbolic spaces. In Ref. [18] the quantum dynamics of scalar particles in SomRaychaudhuri space-time, were investigated and compared the results with the Landau levels in flat space. In Ref. [19] the quantum dynamics of spin-half particles (Dirac fermions) in Som-Raychaudhuri background space-time with torsion and a cosmic string passing through them, were investiagted. They observed that the presence of the topological defect breaks the degeneracy of the relativistic energy levels and the corresponding eigenfunctions depend on the topological defect in the background space-time with torsion. In Ref. [20] the quantum dynamics of spin-half particles (Weyl fermions) in Som-Raychaudhuri space-time with a topological defect, were investigated. In Ref. [21] the scalar quantum particle in a class of Gödel-type solutions with a cosmic string passing through the space-time, were investigated. In Ref. [22] 
the Fermi field and Dirac oscillator in a Som-Raychaudhuri space-time, were investigated. The Klein-Gordon equation with a vector and a scalar potentials of Coulomb-type under the influence of non-inertial effects in the cosmic string space-time, were investigated in Ref. [23]. Two different classes of solutions for the Klein-Gordon equation in the presence of Coulomb-like scalar potential under the influence of non-inertial effects in the cosmic string space-time, were studied in Ref. [24].

Computing the quantities the energy and momentum in curved space-time is still an open problem in general relativity. Following Einstein's original pseudotensor for energymomentum [23], several expressions have been introduced in the literature (see references in Ref. [24]). Various authors evaluated the energy and momentum distributions in different space-times using the known complexes and found quite interesting results. For example, the energy and momentum distributions in $(1+2)$-dimensions rotating BTZ black hole solution [25], non-static circularly symmetric space-time [26], $(1+2)$-dimensions non-rotating black hole solutions with non-zero cosmological constant under various parameter conditions [27], and $(1+2)$-dimensions circularly symmetric Gurses space-time [28]. In four-dimensions spacetime, numerous work have been done by physicists (see Ref. [24] and references therein). In Refs. [29-31] the energy and momentum distributions of a closed homogeneous isotropic universe described by Friedmann-Robertson-Walker (FRW) space-time were evaluated and are equal to zero. The localization of energy and momentum in curved space-times are in doubts. According to Misner et al. [32] the energy is localizable only for spherical systems, whereas for Cooperstock et al. [33] it is for all systems. Cooperstock [34-36] later proposed a hypothesis that the energy and momentum are confined to the region of non-vanishing stress-energy tensor $\left(T_{v}^{\mu}\right)$ of matter and all non-gravitational fields in curved space-time. This hypothesis has neither been proved nor disproved. On the contrary, there are many results which supported this hypothesis (see Ref. [24] and references therein). In the present work, we evaluate the energy and momentum distributions in Som-Raychaudhuri space-time using the known energy-momentum complexes.

In the present work, the quantum dynamics of spin-half particles (Dirac fermions) by solving the Dirac equation in the presence of scalar and vector potentials in SomRaychaudhuri space-time, are study in details. This paper is organized as: in Sect. 2, we solve the Dirac equation in SomRaychaudhuri space-time with scalar and vector potentials and evaluate the energy spectrum and corresponding wave functions using the quasi exactly solvable (QES) method and Nikiforova-Uvarov (NU) method. In addition, we calculate the energy-momentum distributions in this SomRaychaudhuri metric with the known energy-momentum complexes in Sect. 3, and finally conclusions in section end.

\section{The Dirac equation in Som-Raychaudhuri space-time with scalar and vector potential}

The Gödel-type solution with torsion and a topological defect can be written in cylindrical cordinates as the space-time metric [22]

$d s^{2}=-\left(d t+\alpha \Omega r^{2} d \varphi\right)^{2}+d r^{2}+\alpha^{2} r^{2} d \varphi^{2}+d z^{2}$.

The Dirac equation for a free Fermi field $\Psi$ of mass $M$ in curved space-time with scalar and vector potential is given by

$\left[i \gamma^{\mu}\left(\nabla_{\mu}+\right.\right.$ i e $\left.\left.A_{\mu}\right)-(M+S(r))\right] \Psi(r, t)=0$

where in Eq. (2.2) $A_{\mu}=(V(r), 0,0,0), \nabla_{\mu}=\left(\partial_{\mu}+\Gamma_{\mu}\right)$ and $\Gamma_{\mu}$ is the affine connection

$\Gamma_{\mu}=\frac{-1}{8} \omega_{\mu a b}\left[\gamma^{a}, \gamma^{b}\right]$

In Eq. (2.2), $\gamma^{\mu}$ are the Dirac matrices in the flat Minkowski space-time, and $\omega_{\mu a b}$ is the spin connection, given by

$\gamma^{\mu}(x)=e_{a}^{\mu} \gamma^{a}$

$\omega_{\mu a b}=\eta_{a c} e_{v}^{c} e_{b}^{\sigma} \Gamma_{\sigma \mu}^{v}-\eta_{a c} e_{b}^{v} \partial_{\mu} e_{v}^{c}$

where in Eq. (2.5) involves the Christoffel symbols $\Gamma_{i j}^{\mu}$ defined as

$\Gamma_{i j}^{\mu}=\frac{1}{2} g^{\mu \nu}\left\{\frac{\partial g_{v i}}{\partial q^{j}}+\frac{\partial g_{v j}}{\partial q^{i}}-\frac{\partial g_{i j}}{\partial q^{\nu}}\right\}$

and $e_{a}^{\mu}(x)$ are the tetrads relate the curved-space-time coordinates $x^{\mu}$ to the Minkowski space-time variables $x^{a}$ [22]

$$
\begin{aligned}
g^{\mu \nu} & =\eta^{a b} e_{a}^{\mu}(x) e_{b}^{\nu}(x) \\
e_{\mu}^{a} & =\left(\begin{array}{cccc}
1 & 0 & \alpha \Omega r^{2} & 0 \\
0 & 1 & 0 & 0 \\
0 & 0 & \alpha r & 0 \\
0 & 0 & 0 & 1
\end{array}\right), \quad e_{a}^{\mu}=\left(\begin{array}{cccc}
1 & 0 & -\Omega r & 0 \\
0 & 1 & 0 & 0 \\
0 & 0 & \frac{1}{\alpha r} & 0 \\
0 & 0 & 0 & 1
\end{array}\right)
\end{aligned}
$$

where $\eta_{a b}=\operatorname{diag}(-+++)$ is the flat Minkowski space-time metric. Also we can read the metric components directly from Eq. (2.1)

$g_{\mu \nu}=\left(\begin{array}{cccc}-1 & 0 & -\alpha \Omega r^{2} & 0 \\ 0 & 1 & 0 & 0 \\ -\alpha \Omega r^{2} & 0 & \alpha^{2} r^{2}-\alpha^{2} \Omega^{2} r^{4} & 0 \\ 0 & 0 & 0 & 1\end{array}\right)$

The first term in Eq. (2.2) reduces to

$i \gamma^{\mu} \partial_{\mu}=i\left(\begin{array}{cc}\partial_{t} & -r \Omega \sigma^{2} \partial_{t}+\sigma^{1} \partial_{r}+\frac{\sigma^{2}}{r \alpha} \partial_{\varphi}+\sigma^{3} \partial_{z} \\ r \Omega \sigma^{2} \partial_{t}-\sigma^{1} \partial_{r}-\frac{\sigma^{2}}{r \alpha} \partial_{\varphi}-\sigma^{3} \partial_{z} & -\partial_{t}\end{array}\right)$ 
Then from Eq. (2.3), we obtain the affine connection,

$$
\begin{aligned}
& \Gamma_{t}=\left(\begin{array}{cc}
\frac{i \Omega}{2} \sigma^{3} & 0 \\
0 & \frac{i \Omega}{2} \sigma^{3}
\end{array}\right) \\
& \Gamma_{\varphi}=\left(\begin{array}{cc}
\frac{i \alpha}{2}\left(r^{2} \Omega^{2}-1\right) \sigma^{3} & 0 \\
0 & \frac{i \alpha}{2}\left(r^{2} \Omega^{2}-1\right) \sigma^{3}
\end{array}\right) \\
& \Gamma_{r}=O_{4 \times 4}, \Gamma_{z}=O_{4 \times 4}
\end{aligned}
$$

The second term in Eq. (2.2) reduces to

$$
i \gamma^{\mu} \Gamma_{\mu}=i\left(\begin{array}{cc}
\frac{i \Omega}{2} \sigma^{3} & \frac{-i}{2 r} \sigma^{2} \sigma^{3} \\
\frac{i}{2 r} \sigma^{2} \sigma^{3} & \frac{-i \Omega}{2} \sigma^{3}
\end{array}\right)
$$

By selecting the wave function as below:

$$
\Psi(t, r, \varphi, z)=\exp (-i E t+i m \varphi+i k z)\left(\begin{array}{l}
\psi(r) \\
\chi(r)
\end{array}\right)
$$

Substituting various term into the Eq. (2.2) and setting $k=0$, we have two relationships

$$
\begin{aligned}
& \left(E-\frac{\Omega}{2} \sigma^{3}-V(r)-(M+S(r)) \psi(r)\right. \\
& +\left(r \Omega \sigma^{2} V(r)-r \Omega \sigma^{2} E\right. \\
& \left.-\frac{m \sigma^{2}}{r \alpha}+\frac{1}{2 r} \sigma^{2} \sigma^{3}+i \sigma^{1} \frac{\partial}{\partial r}\right) \chi(r)=0 \\
& \left(E-\frac{\Omega}{2} \sigma^{3}-V(r)+(M+S(r)) \chi(r)\right. \\
& +\left(r \Omega \sigma^{2} V(r)-r \Omega \sigma^{2} E\right. \\
& \left.-\frac{m \sigma^{2}}{r \alpha}+\frac{1}{2 r} \sigma^{2} \sigma^{3}+i \sigma^{1} \frac{\partial}{\partial r}\right) \psi(r)=0 .
\end{aligned}
$$

By choosing $V(r)=S(r)$ and substituting $\chi(r)$ from Eq. (2.17) in Eq. (2.16) we obtain

$$
\begin{aligned}
& \psi^{\prime \prime}(r)+\left[E^{2}-M^{2}-2 E \Omega \sigma^{3}\right. \\
& +\frac{\Omega^{2}}{4}-2(M+E) V(r)+2 \Omega \sigma^{3} V(r) \\
& -\frac{2 m}{\alpha} \Omega(E-V(r))+\Omega \sigma^{3} V(r) \\
& -\left(\frac{m^{2}}{\alpha^{2}}-\frac{m}{\alpha} \sigma^{3}+\frac{1}{4}\right) \frac{1}{r^{2}} \\
& \left.-\left(E^{2}+V^{2}(r)-2 E V(r)\right) r^{2} \Omega^{2}\right] \psi(r)=0 .
\end{aligned}
$$

Here we have considered the potentials as follow:
Case 1: $\mathrm{V}(\mathrm{r})=0$

$$
\begin{aligned}
& {\left[\frac{d^{2}}{d r^{2}}-E^{2} \Omega^{2} r^{2}-\frac{\left(\frac{m s}{\alpha}-\frac{1}{2}\right)^{2}}{r^{2}}\right.} \\
& \quad+\left(E^{2}-M^{2}-2 E \Omega s\right. \\
& \left.\left.\quad+\frac{\Omega^{2}}{4}-\frac{2 m E \Omega}{\alpha}\right)\right] \psi(r)=0,
\end{aligned}
$$

where $\sigma^{3} \psi(r)=s \psi(r)$ and $s= \pm 1$, also we can rewrite the differential Eq. (2.19) in a simpler form

$\psi^{\prime \prime}(r)+\left(\lambda_{1} r^{2}+\frac{\lambda_{2}}{r^{2}}+\lambda_{3}\right) \psi(r)=0$

where

$$
\begin{aligned}
& \lambda_{1}=-E^{2} \Omega^{2} \\
& \lambda_{2}=-\left(\frac{m s}{\alpha}-\frac{1}{2}\right)^{2} \\
& \lambda_{3}=E^{2}-M^{2}-2 E \Omega s+\frac{\Omega^{2}}{4}-\frac{2 m \Omega E}{\alpha} .
\end{aligned}
$$

By using the transformation of the form $z=r^{2}$ in Eq. (2.20),we obtain

$\frac{d^{2}}{d z^{2}}+\frac{1}{2 z} \frac{d}{d z}+\frac{1}{z^{2}}\left(\frac{\lambda_{1}}{4} z^{2}+\frac{\lambda_{2}}{4}+\frac{\lambda_{3}}{4} z\right) \psi(z)=0$

If we compare this second-order differential equation with the Nikiforov-Uvarov (NU) form, we obtain the wavefunction

$\Psi(r, t)=r^{\mid\left(\frac{m s}{\alpha}-\frac{1}{2}\right)} e^{\left(-i E t-\frac{|E \Omega|}{2} r^{2}\right)} L_{n}^{\left(\left|\frac{m s}{\alpha}-\frac{1}{2}\right|\right)}\left(|E \Omega| r^{2}\right)$

where $L_{n}^{\alpha}(x)$ is the generalized (or associated) Laguerre polynomial, and so we obtain from Eq. (A.3) in Ref. [22]

$$
\begin{gathered}
2|E \Omega|(2 n+1)-\left(E^{2}-M^{2}-2 E \Omega s+\frac{\Omega^{2}}{4}\right. \\
\left.-\frac{2 m \Omega E}{\alpha}\right)+2|E \Omega|\left|\left(\frac{m s}{\alpha}-\frac{1}{2}\right)\right|=0
\end{gathered}
$$

For $E>0$, the energy for Eq. (2.24) is given by

$$
\begin{aligned}
& E_{n, m}=\frac{1}{2 \alpha}[2 m \Omega(s+1)+\alpha \Omega(1+4 n+2 s) \\
& \left.+\sqrt{(2 m(s+1)+\alpha(1+4 n+2 s))^{2} \Omega^{2}+\alpha^{2}\left(4 M^{2}-\Omega^{2}\right)}\right]
\end{aligned}
$$


For $m=0$, the energy eigenvalue reduces to

$$
\begin{aligned}
E_{n, 0}= & \frac{1}{2 \alpha}[\alpha \Omega(1+4 n+2 s) \\
& \left.+\sqrt{\alpha^{2}(1+4 n+2 s)^{2} \Omega^{2}+\alpha^{2}\left(4 M^{2}-\Omega^{2}\right)}\right]
\end{aligned}
$$

For $E<0$, the energy for Eq. (2.24) is given by

$$
\begin{aligned}
& E_{n, m}=\frac{1}{2 \alpha}[2 m \Omega(s-1)-\alpha \Omega(1+4 n+2 s) \\
& \left.\quad+\sqrt{(2 m(s-1)-\alpha(1+4 n+2 s))^{2} \Omega^{2}+\alpha^{2}\left(4 M^{2}-\Omega^{2}\right)}\right]
\end{aligned}
$$

For $m=0$, the energy eigenvalue reduces to

$$
\begin{aligned}
E_{n, 0}= & \frac{1}{2 \alpha}[-\alpha \Omega(1+4 n+2 s) \\
& \left.+\sqrt{\left(\alpha^{2}(1+4 n+2 s)^{2} \Omega^{2}+\alpha^{2}\left(4 M^{2}-\Omega^{2}\right)\right.}\right]
\end{aligned}
$$

Case 2: $V(r)=\frac{a}{r}$

By introducing the Coulomb scalar potential $V(r)=\frac{a}{r}$ where $a$ is constant, we obtain

$$
\begin{aligned}
H & =\frac{d^{2}}{d r^{2}}-E^{2} \Omega^{2} r^{2}+2 E \Omega^{2} a r \\
& +\left(E^{2}-M^{2}-2 E \Omega s+\frac{\Omega^{2}}{4}\right. \\
& \left.+\frac{2 m}{\alpha} E \Omega-\Omega^{2} a^{2}\right) \\
& +\left\{(2 \Omega+1) s a+\frac{2 m}{\alpha} a \Omega-2(M+E) a\right\} \frac{1}{r} \\
& +\left(\frac{m}{\alpha} s-\frac{m^{2}}{\alpha^{2}}+\frac{1}{4}\right) \frac{1}{r^{2}} .
\end{aligned}
$$

Making the gauge transformation

$\psi(r)=G \tilde{\psi}(r)$

where is $G=e^{-A}$ and A obtained from Eq. (A.9) and Table 1 in Ref. [37]

$$
\begin{aligned}
A= & {\left[\Omega a r+\frac{E \Omega r^{2}}{2}\right.} \\
& \left.-\frac{1}{2}\left\{1-\sqrt{1+4\left(\frac{m s}{\alpha}-\frac{1}{2}\right)^{2}}\right\} \ln r\right] .
\end{aligned}
$$

In this case, $\tilde{H}$ is equivalent to the $H$ under the transformation given by

$$
\tilde{H}=G^{-1} H G
$$

From Eq. (2.32) we obtain

$$
\begin{aligned}
\tilde{H} \equiv & r \frac{d^{2}}{d r^{2}}+\left[2 \Omega a r-2 E \Omega r^{2}-1\right. \\
& +\sqrt{\left.1+4\left(\frac{m s}{\alpha}-\frac{1}{2}\right)^{2}\right]} \frac{d}{d r} \\
& +\left[E \Omega+\Omega^{2} a^{2}\right. \\
& +E \Omega\left\{-1+\sqrt{1+4\left(\frac{m s}{\alpha}-\frac{1}{2}\right)^{2}}\right\}+E^{2}-M^{2} \\
& \left.-2 E \Omega s+\frac{\Omega^{2}}{4}+\frac{2 m}{\alpha} E \Omega-\Omega^{2} a^{2}\right] r-2 E \Omega^{2} a \\
& +2 \frac{m}{\alpha} a \Omega+a s \\
& -\Omega a\left\{-1+\sqrt{1+4\left(\frac{m s}{\alpha}-\frac{1}{2}\right)^{2}}\right\} \\
& +2 \Omega a s-2(E+M) a
\end{aligned}
$$

From Eq. (A.14), any QES differential operator can be represented a

$$
\begin{aligned}
\tilde{H}= & C_{++} J_{n}^{+} J_{n}^{+}+C_{+0} J_{n}^{+} J_{n}^{0} \\
& +C_{+-} J_{n}^{+} J_{n}^{-}+C_{0-} J_{n}^{0} J_{n}^{-}+C_{--} J_{n}^{-} J_{n}^{-} \\
& +C_{+} J_{n}^{+}+C_{0} J_{n}^{0}+C_{-} J_{n}^{-}+C,
\end{aligned}
$$

where

$$
\begin{aligned}
& J_{n}^{+}=r^{2} \frac{d}{d r}-n r \\
& J_{n}^{0}=r \frac{d}{d r}-\frac{n}{2}, \\
& J_{n}^{-}=\frac{d}{d r} .
\end{aligned}
$$

Substitution of Eq. (2.35) into Eq. (2.34) yields the following differential for

$$
\left(P_{4} \frac{d^{2}}{d r^{2}}+P_{3} \frac{d}{d r}+P_{2}\right) \tilde{\Psi}(r)=0
$$

where $P_{i}$ are the polynomials of degree i;

$$
\begin{aligned}
P_{4}= & C_{++} r^{4}+C_{+0} r^{3}+C_{+-} r^{2}+C_{0-} r+C_{--}, \\
P_{3}= & C_{++}(2-2 n) r^{3}+\left(C_{+}+C_{+0}\left(1-\frac{3 n}{2}\right)\right) r^{2} \\
& +\left(C_{0}-n C_{+-}\right) r+\left(C_{-}-\frac{n}{2} C_{0-}\right), \\
P_{2}= & C_{++} n(n-1) r^{2}+\left(\frac{n^{2}}{2} C_{+0}-n C_{+}\right) r \\
& +\left(C-\frac{n}{2} C_{0}\right) .
\end{aligned}
$$


Here the coefficients $C_{++}, \ldots$ in the polynomials $P_{i}$ are

$$
\begin{aligned}
& C_{++}=C_{+0}=C_{+-}=C_{--}=0, \\
& C_{0-}=1, \quad C_{+}=-2 E \Omega, \\
& C_{0}=2 \Omega a, \quad C_{-}=-1+\sqrt{1+4\left(\frac{m s}{\alpha}-\frac{1}{2}\right)^{2}+\frac{n}{2},} \\
& C=\frac{n}{2} C_{0}-2 E \Omega^{2} a-\Omega a\left\{-1+\sqrt{1+4\left(\frac{m s}{\alpha}-\frac{1}{2}\right)^{2}}\right\} \\
& +\frac{2 m}{\alpha} \Omega+a s+2 \Omega a s-2(M+E) a
\end{aligned}
$$

Therefore we have the following eigenvalue equations using Eq. (2.38):

$$
\begin{aligned}
-n C_{+}= & 2 \Omega E+E \Omega\left(-1+\sqrt{1+4\left(\frac{m s}{\alpha}-\frac{1}{2}\right)^{2}}\right) \\
& +E^{2}-M^{2}-2 E \Omega s+\frac{\Omega^{2}}{4}+\frac{2 m}{\alpha} E \Omega
\end{aligned}
$$

Substituting Eq. (2.38) into the Eq. (2.39), we get the following energy eigenvalues : where $\tilde{\psi}(r)$ is:

$\tilde{\psi}(r)=\sum_{n=0}^{\infty} a_{n} r^{n}$

For $n=0$ the eigenfunction has the form $\tilde{\psi}(r)=a_{0}$ and the corresponding matrix equation is given by

$v a_{0}=0$

where

$v=2 \Omega a s+a s-2 a(M+E)+\frac{2 m}{\alpha} a \Omega-2 E a \Omega^{2}$

$$
-\Omega a\left(-1+\sqrt{1+4\left(\frac{m s}{\alpha}-\frac{1}{2}\right)^{2}}\right)
$$

Thus, from Eqs. (2.30) and (2.31) and (2.15), we obtain the ground-state wavefunction as

$$
\begin{aligned}
& E_{n, m}=\frac{1}{2 \alpha}\left(-2 m \Omega-\alpha \Omega\left(1-2 s-2 n+\sqrt{1+4\left(\frac{m s}{\alpha}-\frac{1}{2}\right)^{2}}\right)\right. \\
& \pm \sqrt{\left(2 m-\alpha\left(1+2 s+2 n-\sqrt{\left.\left.1+4\left(\frac{m s}{\alpha}-\frac{1}{2}\right)^{2}\right)\right)^{2} \Omega^{2}+\alpha^{2}\left(4 M^{2}-\Omega^{2}\right)}\right)\right.}
\end{aligned}
$$

For $m=0$, the energy eigenvalue reduces to

$$
\begin{aligned}
& E_{n, 0}=\frac{1}{2 \alpha}(-\alpha \Omega(1-2 s-2 n+\sqrt{2}) \pm \\
& \left.\sqrt{(-\alpha \Omega(1+2 s+2 n-\sqrt{2}))^{2}+\alpha^{2}\left(4 M^{2}-\Omega^{2}\right)}\right)
\end{aligned}
$$

With putting Eqs. (2.35) and (2.38) in Eq. (2.34):

$$
\begin{aligned}
\tilde{H}= & r \frac{d^{2}}{d r^{2}}+\left(2 \Omega a r-2 E \Omega r^{2}-1\right. \\
& +\frac{n}{2}+\sqrt{\left.1+4\left(\frac{m s}{\alpha}-\frac{1}{2}\right)^{2}\right)} \frac{d}{d r} \\
& +2 n E \Omega r+2 \Omega s a+s a-2(M-E) a \\
& +\frac{2 m a \Omega}{\alpha}-2 E a \Omega^{2} \\
& -a \Omega\left(-1+\sqrt{1+4\left(\frac{m s}{\alpha}-\frac{1}{2}\right)^{2}}\right.
\end{aligned}
$$

$$
\begin{aligned}
\Psi_{0}(r, t)= & \exp \left(-i E t-\Omega a r-\frac{E \Omega r^{2}}{2}\right. \\
& \left.+\left(\frac{1}{2}-\frac{1}{2} \sqrt{1+4\left(\frac{m s}{\alpha}-\frac{1}{2}\right)^{2}}\right) \ln r\right)
\end{aligned}
$$

For $n=1$ the corresponding eigenfunction becomes $\tilde{\psi}(r)=a_{0}+a_{1} r$. The corresponding matrix equation is therefore given by

$$
\left(\begin{array}{cc}
v & -1+\sqrt{1+4\left(\frac{m s}{\alpha}-\frac{1}{2}\right)^{2}} \\
2 E \Omega & v+2 a \Omega
\end{array}\right)\left(\begin{array}{l}
a_{0} \\
a_{1}
\end{array}\right)=0
$$

From Eq. (2.4), we obtain

$$
a_{1}=\frac{v}{\sqrt{1+4\left(\frac{m s}{\alpha}-\frac{1}{2}\right)^{2}}-1} a_{0}
$$

Therefore, by using Eqs. (2.30) and (2.31) and (2.15), the wavefunction of the first excited-state can be written as 


$$
\begin{aligned}
& \Psi_{1}(r, t)=\exp \left[-i E t-\Omega a r-\frac{E \Omega r^{2}}{2}\right. \\
& \left.+\left(\frac{1}{2}-\frac{1}{2} \sqrt{1+4\left(\frac{m s}{\alpha}-\frac{1}{2}\right)^{2}}\right) \ln r\right] \\
& \times\left(1+\frac{v}{\sqrt{1+4\left(\frac{m s}{\alpha}-\frac{1}{2}\right)^{2}}-1} r\right)
\end{aligned}
$$

\section{Energy-momentum}

Energy-momentum localization plays a leading role in the theories advanced over the years in relation to general relativity. There is not a satisfactory description for the gravitational energy and for formulating a proper definition for the energy density of gravitational backgrounds there is a major difficulty. In this section, we try to evaluate the energy and momentum distributions in the Som-Raychaudhuri spacetime Eq. (2.1) using the known pseudotensor, the LandauLifshitz complex, the Einstein complex, and the Papapetrou complex [30,38-40].

\subsection{Landau-Lifshitz energy-momentum}

The energy and momentum densities in Landau-Lifshitz [30, $38,41]$ complex is given by

$$
\begin{aligned}
L^{\mu \rho} & =\frac{1}{16 \pi} S_{, \nu \sigma}^{\mu \nu \rho \sigma}, \\
S^{\mu \nu \rho \sigma} & =-g\left(g^{\mu \rho} g^{\nu \sigma}-g^{\mu \sigma} g^{\nu \rho}\right),
\end{aligned}
$$

where $g$ is $\operatorname{det}\left(g_{\mu \nu}\right)$ and the non-zero components of $S^{\mu \nu \rho \sigma}$ are

$$
\begin{aligned}
& S^{0101}=\alpha^{2} r^{2}\left(r^{2} \Omega^{2}-1\right), \\
& S^{0202}=\alpha^{2} r^{2}\left(\frac{r^{2} \Omega^{2}-1}{\alpha^{2} r^{2}}-\frac{\Omega^{2}}{\alpha^{2}}\right), \\
& S^{0303}=\alpha^{2} r^{2}\left(r^{2} \Omega^{2}-1\right) .
\end{aligned}
$$

Substituting Eq. (3.3) in Eq. (3.1), we get the energydensity

$L^{00}=\frac{1}{16 \pi}\left(S_{, 11}^{0101}+S_{, 22}^{0202}+S_{, 33}^{0303}\right)=\frac{1}{8 \pi}\left(6 \alpha^{2} r^{2} \Omega^{2}-\alpha^{2}\right)$,

Therefore the energy which is contained in a Cylinder of radisu $r$ and length $L$ (since the spatial section of the $(1+$ 3)-dimensional space-time is Three-dimensional the a oneCylinder ) given by

$E_{L}=\int_{0}^{r} \int_{0}^{2 \pi} \int_{0}^{L} L^{00} r d r d \varphi d z=\frac{L}{8} \alpha^{2} r^{2}\left(3 \Omega^{2} r^{2}-1\right)$.

\subsection{Einstein energy-momentum}

The energy-momentum complex is defined by $[25,30,38]$

$\Theta_{\mu}^{v}=\frac{1}{16 \pi} H_{\mu, \rho}^{v \rho}$

where $H_{\mu}^{v \rho}$ are

$H_{\mu}^{\nu \rho}=-H_{\mu}^{\rho \nu}=\frac{g_{\mu \tau}}{\sqrt{-g}}\left[-g\left(g^{\nu \tau} g^{\rho \sigma}-g^{\rho \tau} g^{\nu \sigma}\right)\right]_{, \sigma}$

$H_{0}^{01}=-2 \alpha \Omega^{2} r^{2}+2 \alpha, H_{0}^{02}=H_{0}^{03}=0$.

Substituting Eq. (3.8) in Eq. (3.6), we get the energy-density

$\Theta_{0}^{0}=\frac{1}{16 \pi} H_{0, \rho}^{0 \rho}=\frac{1}{16 \pi}\left(H_{0,1}^{01}+H_{0,2}^{02}+H_{0,3}^{03}\right)=\frac{-1}{4 \pi}\left(\alpha \Omega^{2} r\right)$

Therefore the energy which is contained in a Cylinder of radius $r$ and length $L$ (since the spatial section of the $(1+$ 3)-dimensional space-time is Three-dimensional the a oneCylinder ) given by

$E_{E}=\int_{0}^{r} \int_{0}^{2 \pi} \int_{0}^{L} \Theta_{0}^{0} r d r d \varphi d z=\frac{-L}{6}\left(\alpha \Omega^{2} r^{3}\right)$

\subsection{Papapetrou energy-momentum}

The symmetric energy-momentum complex of Papapetrou $[30,38,42]$ is given as

$\Omega^{\mu \nu}=\frac{1}{16 \pi} N_{, \rho \sigma}^{\mu \nu \rho \sigma}$

where $N^{\mu \nu \rho \sigma}$ are

$N^{\mu \nu \rho \sigma}=\sqrt{-g}\left(g^{\mu v} \eta^{\rho \sigma}-g^{\mu \rho} \eta^{\nu \sigma}+g^{\rho \sigma} \eta^{\mu \nu}-g^{\nu \sigma} \eta^{\mu \rho}\right)$

$N^{0011}=\left(\alpha \Omega^{2} r^{3}-2 \alpha r\right), \quad N^{0022}=\alpha r\left(r^{2} \Omega^{2}+\frac{1}{\alpha^{2} r^{2}-1}\right)$, $N^{0033}=\alpha r^{3} \Omega^{2}$.

Substituting Eq. (3.13) in Eq. (3.11), we get the energydensity

$\Omega^{00}=\frac{1}{16 \pi} N_{, \rho \sigma}^{00 \rho \sigma}=\frac{1}{16 \pi}\left(N_{, 11}^{0011}+N_{, 22}^{0022}+N_{, 33}^{0033}\right)=\frac{3}{8 \pi} \alpha \Omega^{2} r$

Therefore the energy which is contained in a Cylinder of radius $r$ and length $L$ (since the spatial section of the $(1+$ 
3)-dimensional space-time is Three-dimensional the a oneCylinder ) given by

$E_{p}=\int_{0}^{r} \int_{0}^{2 \pi} \int_{0}^{L} \Omega^{00} r d r d \varphi d z=\frac{L}{4}\left(\alpha \Omega^{2} r^{3}\right)$

Notice that from Eqs. (3.5) and (3.10) and (3.15) we can write

$E_{p}=-\frac{3}{2} E_{E}=-\frac{2}{3 \alpha r} E_{L}+\frac{1}{6} \alpha r$.

\section{Conclusion}

We have studied the behaviour of Dirac fermions in a class of Gödel background space-times with a topological defect. We have solved the Dirac equation under scalar and vector potential in Som-Raychaudhuri space-time by quasi-exactly solvable (QES) differential equations method and NU method. We have evaluated the energy eigenvalues in the presence of this $V(r)=0$ potential and coulomb potential $V(r)=\frac{a}{r}$ and shown that the energy spectrum dependent in a SomRaychaudhuri space-time to $\Omega$ and breaks the degeneracy of the energy levels. In Sect. 3, we have calculated the energy and momentum distributions in Som-Raychaudhuri spacetime using the known complexes, the Einstein's, the LandauLifshitz's, and the Papapetrou energy-momentum complex.

Acknowledgements The authors thank the referees for a thorough reading of our manuscript and for constructive suggestions.

Data Availability Statement This manuscript has no associated data or the data will not be deposited. [Authors' comment: As we have don't any numerical results therefore we don't use them.]

Open Access This article is distributed under the terms of the Creative Commons Attribution 4.0 International License (http://creativecomm ons.org/licenses/by/4.0/), which permits unrestricted use, distribution, and reproduction in any medium, provided you give appropriate credit to the original author(s) and the source, provide a link to the Creative Commons license, and indicate if changes were made.

Funded by SCOAP ${ }^{3}$.

\section{Appendix}

Quasi-exactly solvable differential equations

A linear differential operator $\mathrm{H}$ in a Hilbert space is called QES [37,43-58].

$$
\begin{aligned}
& (H-E) \Psi=0 \\
& H_{0}=H-E \\
& H_{0} \Psi=0
\end{aligned}
$$

$\tilde{H}$ is equivalent to the $H_{0}$ under the transformation given by
$\tilde{H} \tilde{\Psi}=0$

Making the gauge [52]

$$
\begin{aligned}
& \tilde{H}=G^{-1} \cdot H \cdot G \\
& G=e^{-A} \\
& \left(P_{4} \frac{d^{2}}{d r^{2}}+P_{3} \frac{d}{d r}+P_{2}\right) \tilde{\Psi}(r)=0 \\
& V(r)=A^{\prime 2}-A^{\prime \prime}+P_{2}(r)
\end{aligned}
$$

where $A$ :

$$
\begin{aligned}
& A=\int \frac{P_{3}}{P_{4}} d r-\log \left(Z^{\prime}\right) \\
& Z=\int \frac{d r}{\sqrt{P_{4}}}
\end{aligned}
$$

In one dimension, the only Lie algebra of the first-order differential operators, which possesses finite-dimensional representations, is the $\mathrm{sl}(2)$ algebra, whose generators

$J_{n}^{+}=r^{2} \frac{d}{d r}-n r$

$J_{n}^{0}=r \frac{d}{d r}-\frac{n}{2}$

$J_{n}^{-}=\frac{d}{d r}$

The operator $\tilde{H}$ can be represented as a quadratic combination of the $\mathrm{sl}(2)$ generators as

$\tilde{H}=\sum_{a, b=0, \pm} C_{a b} J^{a} J^{b}+\sum_{a=0, \pm} C_{a} J^{a}+C$

any QES differential operator can be represented as

$$
\begin{aligned}
\tilde{H}= & C_{++} J_{n}^{+} J_{n}^{+}+C_{+0} J_{n}^{+} J_{n}^{0}+C_{+-} J_{n}^{+} J_{n}^{-}+C_{0-} J_{n}^{0} J_{n}^{-} \\
& +C_{--} J_{n}^{-} J_{n}^{-}+C_{+} J_{n}^{+}+C_{0} J_{n}^{0}+C_{-} J_{n}^{-}+C
\end{aligned}
$$

Substitution of Eqs. (A.11-A.13) into Eq. (A.15) yields the following differential form:

$$
\left(P_{4} \frac{d^{2}}{d r^{2}}+P_{3} \frac{d}{d r}+P_{2}\right) \tilde{\Psi}(r)=0
$$

where $\mathrm{Pi}$ are the polynomials of degree $\mathrm{i}$;

$$
\begin{aligned}
P_{4}= & C_{++} r^{4}+C_{+0} r^{3}+C_{+-} r^{2}+C_{0-} r+C_{--} \\
P_{3}= & C_{++}(2-2 n) r^{3}+\left(C_{+}+C_{+0}\left(1-\frac{3 n}{2}\right)\right) r^{2} \\
& +\left(C_{0}-n C_{+-}\right) r+\left(C_{-}-\frac{n}{2} C_{0-}\right) \\
P_{2}= & C_{++} n(n-1) r^{2}+\left(\frac{n^{2}}{2} C_{+0}-n C_{+}\right) r \\
& +\left(C-\frac{n}{2} C_{0}\right)
\end{aligned}
$$


with comparing Eqs. (A.16-A.19) we find $C_{++}, C_{--}, .$. and in end

$$
\begin{aligned}
\tilde{\Psi} & =\sum_{m=0} a_{m} r^{m} \\
\Psi & =G \cdot \tilde{\Psi}
\end{aligned}
$$

\section{References}

1. M.M. Som, A.K. Raychaudhuri, Proc. R. Soc. A 304, 81 (1968)

2. J.D. Barrow, M.P. Dabrowski, Phys. Rev. D 58, 103502 (1998)

3. O. Bertolami, F. Lobo, Neuro Quantol. 7, 1 (2009)

4. P. Kanti, C.E. Vayonakis, Phys. Rev. D 60, 103519 (1999)

5. J.D. Barrow, C. Tsagas, Class. Quantum Grav. 21, 1773 (2004)

6. J.D. Barrow, C. Tsagas, Phys. Rev. D 69, 064007 (2004)

7. F.M. Paiva, M.J. Reboucas, A.F.F. Teixeira, Phys. Lett. A 126, 168 (1987)

8. R.J. Gleiser, M. Gürses, A. Karasu, S. Ozgur, Class. Quantum Grav. 23, 2653 (2006)

9. M. Gürses, A. Karasu, Ö. Sarioğlu, Class. Quantum Grav. 22, 1527 (2018)

10. T. Clifton, J. Barrow, Phys. Rev. D 72, 123003 (2005)

11. Z. Wang, Z. Long, C. Long, M. Wu, EPJ Plus 130, 36 (2015)

12. J. Carvalho, A.M. de M. Carvalho, C. Furtado, EPJ C 74, 2935 (2014)

13. R.L.L. Vitoria, C. Furtado, K. Bakke, EPJ C 78, 44 (2018)

14. F. Ahmed, EPJ C 79(02), 104 (2019)

15. B.D.B. Figueiredo, I.D. Soares, J. Tiomno, Class. Quantum Grav. 9, 1593 (1992)

16. N. Drukker, B. Fiol, J. Simon, JCAP 0410, 012 (2004)

17. N. Drukker, B. Fiol, J. Simon, Phys. Rev. Lett. 91, 231601 (2003)

18. S. Das, J. Gegenberg, Gen. Relativ. Gravit. 40, 2115 (2008)

19. G.Q. Garcia, J.R. de S. Oliveira, K. Bakke, C. Furtado, EPJ Plus 132, 123 (2017)

20. G.Q. Garcia, J.R. de S. Oliveira, C. Furtado, Int. J. Mod. Phys. 27, 1850027 (2018)

21. J. Carvalho, A.M. de M. Carvalho, E. Cavalcante, C. Furtado, EPJ C 76, 365 (2016)

22. M. de Montigny, S. Zare, H. Hassanabadi, Gen. Relativ. Gravit. 50, $47(2018)$

23. L.C.N. Santos, C.C. Barros Jr., EPJ C 78, 13 (2018)

24. L.C.N. Santos, C.C. Barros Jr., EPJ C 77, 186 (2017)
25. A. Trautman, in Gravitation: An Introduction to Current Research, ed. by L. Witten (Wiley, New York, 1962)

26. F. Ahmed, EPJ C 78, 598 (2018)

27. Elias C. Vagenas, Int. J. Mod. Phys. A 18, 5949 (2003)

28. Elias C. Vagenas, Int. J. Mod. Phys. D 14, 573 (2005)

29. I.C. Yang, I. Radinschi, AIP Conf. Proc. 895, 325 (2007)

30. F. Ahmed, Ann. Phys. (N. Y.) 401, 193 (2019)

31. N. Rosen, Gen. Relativ. Gravit. 26, 319 (1994)

32. C.W. Misner, K.S. Thorne, J.A. Wheeler, Gravitation (W. H. Freeman and Co., New York, 1973)

33. F.I. Cooperstock, Gen. Relativ. Gravit. 26, 323 (1994)

34. F.I. Cooperstock, R.S. Sarracino, J. Phys. A 11, 877 (1978)

35. F.I. Cooperstock, Found. Phys. 22, 1011 (1992)

36. V.B. Johri, D. Kalligas, G.P. Singh, C.W.F. Everitt, Gen. Relativ. Gravit. 27, 313 (1995)

37. A.V. Turbiner, Commun. Math. Phys. 118, 467 (1988)

38. C. Furtado, B.C.G. da Cunha, F. Moraes, E.R. Bezerra de Mello, V.B. Bezerra, Phys. Lett. A 195, 90 (1994)

39. S. Weinberg, Gravitation and Cosmology: Principles and Applications of General Theory of Relativity (Wiley, New York, 1972)

40. I. Radinschi, F. Rahaman, T. Grammenos, S. Islam, Adv. High Energy Phys. 2016, 9049308 (2016)

41. L.D. Landau, E.M. Lifshitz, The Classical Theory of Fields (Pergamon Press, New York, 1987)

42. A. Papapetrou, Proc. R. Ir. Acad. A 52, 11 (1948)

43. A.V. Turbiner, Contemp. Math. 160, 263 (1994)

44. D. Gomez-Ullate, N. Kamran, R. Milson, Phys. At. Nucl. 70, 520 (2007)

45. A.G. Ushveridze, Mod. Phys. Lett. A 5, 1891 (1990)

46. Y.Z. Zhang, J. Math. Phys. 54, 102104 (2013)

47. Y.H. Lee, J. Links, Y.Z. Zhang, J. Phys. A Math. Theor. 44, 482001 (2011)

48. R. Sasaki, J. Math. Phys. 48, 122104 (2007)

49. C.L. Ho, Ann. Phys. 321, 2170 (2006)

50. H. Panahi, M. Baradaran, Mod. Phys. Lett. A 27, 1250176 (2012)

51. H. Panahi, M. Baradaran, Eur. Phys. J. Plus 128, 39 (2013)

52. H. Panahi, S. Zarrinkamar, M. Baradaran, Chin. Phys. B 24(6), 060301 (2015)

53. F.M. Fernandez, Phys. Lett. A 166, 173 (1992)

54. R.K. Roychoudhury, Y.P. Varshni, M. Sengupta, Phys. Rev. A 42, 184 (1990)

55. R.N. Chaudhuri, J. Phys. A Math. Gen. 21, 567 (1988)

56. G. Plante, A.F. Antippa, J. Math. Phys. 46, 062108 (2005)

57. H. Weyl, The Theory of Groups and Quantum Mechanics (Dover, New York, 1931)

58. R.P. Saxena, V.S. Varma, J. Phys. A Math. Gen. 15, L221 (1982) 